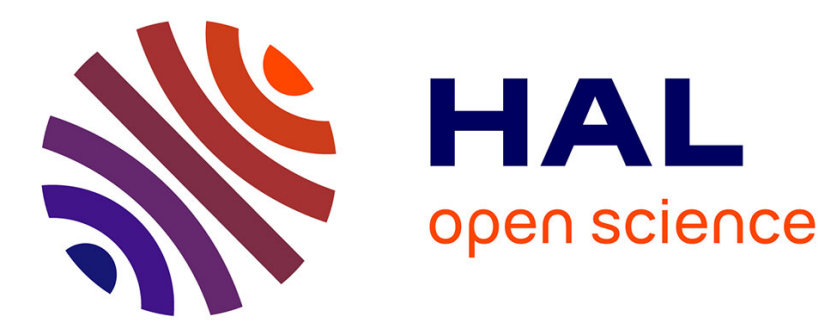

\title{
Stoneley-type waves in anisotropic periodic superlattices
}

A.N. Darinskii, A.L. Shuvalov

\section{To cite this version:}

A.N. Darinskii, A.L. Shuvalov. Stoneley-type waves in anisotropic periodic superlattices. Ultrasonics, 2021, 109, pp.106237. 10.1016/j.ultras.2020.106237 . hal-03381745

\section{HAL Id: hal-03381745 \\ https://hal.science/hal-03381745}

Submitted on 22 Oct 2021

HAL is a multi-disciplinary open access archive for the deposit and dissemination of scientific research documents, whether they are published or not. The documents may come from teaching and research institutions in France or abroad, or from public or private research centers.
L'archive ouverte pluridisciplinaire HAL, est destinée au dépôt et à la diffusion de documents scientifiques de niveau recherche, publiés ou non, émanant des établissements d'enseignement et de recherche français ou étrangers, des laboratoires publics ou privés. 


\title{
Stoneley-type waves in anisotropic periodic superlattices
}

\author{
A.N. Darinskii ${ }^{a, *}$, A.L. Shuvalov ${ }^{\mathrm{b}}$ \\ a Institute of Crystallography FSRC “Crystallography and Photonics”, Russian Academy of Sciences, Leninskii pr. 59, Moscow 119333, Russia \\ ${ }^{\mathrm{b}}$ Univ. Bordeaux, CNRS, Bordeaux INP, Arts et Metiers Institute of Technology, I2M Bordeaux, UMR 5295, F-33405 Talence, France
}

A R T I C L E I N F O

\section{Keywords:}

Interfacial acoustic waves

Periodic superlattice

Elastic anisotropy

Transfer matrix

Impedance

\begin{abstract}
A B S T R A C T
The paper investigates the existence of interfacial (Stoneley-type) acoustic waves localised at the internal boundary between two semi-infinite superlattices which are adjoined with each other to form one-dimensional phononic bicrystal. Each superlattice is a periodic sequence of perfectly bonded homogeneous and/or functionally graded layers of general anisotropy. Given any asymmetric arrangement of unit cells (periods) of superlattices, it is found that the maximum number of interfacial waves, which can emerge at a fixed tangential wavenumber for the frequency varying within a stopband, is three for the lowest stopband and six for any upper stopband. Moreover, we show that this number of three or six waves in the lowest or upper stopband, is actually the maximum for the number of waves occurring per stopband in a given bicrystal plus their number in the "complementary" bicrystal, which is obtained by swapping upper and lower superlattices of the initial one (so that both bicrystals have the same band structure). An example is provided demonstrating attainability of this upper bound, i.e. the existence of six interfacial waves in a stopband. The results obtained under no assumptions regarding the material anisotropy are also specified to the case of monoclinic symmetry leading to acoustic mode decoupling.
\end{abstract}

\section{Introduction}

Surface and interfacial acoustic wave solutions normally come about within such ranges of frequency $\omega$ and wave vector $\mathbf{k}$ which preclude existence of the propagating partial modes, so that the boundary condition can be satisfied by a wave packet that wholly consists of modes decaying into the depth of surrounding media and thus is localised near the guiding boundary. Localised waves in homogeneous halfspaces, e.g. the Rayleigh and Stoneley waves, are non-dispersive and typically exist in the subsonic velocity interval extending from zero up to a certain threshold velocity [1]. By contrast, localised waves in periodic halfspaces are dispersive and may exist inside the infinite sequence of subdomains of the $(\omega, \mathbf{k})$ space called forbidden bands or stopbands [2-4]. Thus the realm of localised waves occurring in periodic media is certainly much more diverse than that in homogeneous materials. Much work has been done in this direction. The largest amount of results has been obtained on surface [5-16] and interfacial [17-21] waves in one-dimensionally periodic structures, or superlattices, in which case analytical developments can be advanced further and the numerical calculations are not that costly as compared to the structures with two- and three-dimensional periodicity. At the same time, no general and rigorous knowledge on the existence of localised waves in anisotropic superlattices can be gained by way of explicit derivations, which do not provide a closed-form dependence on the involved parameters even in the simplest case of isotropic materials; neither can it be extrapolated from numerical results, which are certainly incapable of embracing infinite variety of possible input data. In this regard, it is clear that an appropriate approach to the question of existence of localised waves should capture the essence of the problem without a need to find these wave solutions themselves.

An efficient approach to surface and interfacial waves in homogeneous anisotropic half-spaces has been developed and applied in the seminal papers [22-29]. It is based on the Stroh formalism and the concept of surface impedance matrix, whose powerful properties follow from its link to energetic quantities. Recently we have applied an extension of this approach coupled with the Floquet-Bloch concepts to the problem of existence of surface waves in elastic and piezoelectric superlattices (1D phononic crystals) of general anisotropy [30-33]. In particular, the analysis reveals that the maximum possible number of surface waves per stopband essentially depends on whether the unit cell of a superlattice is asymmetric or symmetric relative to its midplane (note that the symmetry in this context concerns the ordering of constituent layers and has nothing to do with their crystallographic symmetry which may be as low as triclinic). Existence of interfacial waves

\footnotetext{
* Corresponding author.

E-mail address: alexandre_dar@mail.ru (A.N. Darinskii).
} 
in 1D phononic bicrystals formed by two superlattices with a symmetric unit cell each was studied in [34].

The present paper is concerned with the most general case of interfacial waves in 1D phononic bicrystals, i.e. of the waves localised at the interface between two perfectly bonded half-infinite superlattices. Each superlattice is periodically layered, possibly functionally graded, medium of arbitrary elastic anisotropy with an arbitrary piecewise constant or continuous variation of material properties within the unit cell (period). It appears that asymmetry of unit cells much ramifies possible scenarios of the occurrence of interfacial waves and eventually augments their possible maximum number allowed at fixed tangential wavenumber per stopband frequency range. It is found that a bicrystal with any asymmetric unit cells admits up to three interfacial waves in the lowest and up to six waves in any upper stopband. This is in contrast to at most one and three waves which may exist in the lowest and upper stopbands of a bicrystal with both halves having a symmetric unit cell [34]. Moreover, the aforementioned bound, which is three or six waves per stopband, is shown to actually be the maximum for the total number of waves occurring per the same stopband in a given bicrystal and in the "complementary" bicrystal, which is obtained by swapping upper and lower superlattices of the initial one. By way of appropriate examples, it is confirmed that the upper bound of the number of interfacial waves per stopband is attainable, i.e., that the number of waves is less or equal (not just less) than the established bound and so this upper bound is a maximum in the formal meaning of it.

The paper has the following structure. The properties of the transfer and impedance matrices are outlined in Section 2. The existence and number of interfacial waves is analysed in Section 3. Numerical examples are presented in Section 4 and the results obtained are summarized in Section 5.

\section{Transfer and impedance matrices}

\subsection{Transfer matrix}

Consider a solid multilayered medium whose density $\rho$ and stiffness tensor $\widehat{\mathbf{c}}=\left(c_{i j k l}\right)$ vary along the stratification axis $Y$ normal to the perfectly bonded layer interfaces. The layers may be homogeneous and/or functionally graded. Assume a displacement wave of the form

$\mathbf{u}(\mathbf{r}, t)=\mathbf{a}(y) \mathrm{e}^{i(k x-\omega t)}$,

where $k$ and $\omega$ are real wave number and frequency, $x=\mathbf{m} \cdot \mathbf{r}$ and $y=\mathbf{n} \cdot \mathbf{r}$ with $\mathbf{m}$ and $\mathbf{n}$ being unit vectors parallel and orthogonal to the plane of interfaces, respectively. The Stroh formalism [35,36] casts the governing equations of elastodynamics in the form of a system

$\frac{d \boldsymbol{\xi}}{d y}=i \widehat{\mathbf{N}} \boldsymbol{\xi}$

with the vector of unknowns

$\boldsymbol{\xi}(y)=(\mathbf{a}(y) \mathbf{l}(y))^{T}$,

which incorporates amplitudes of the displacement and of the normal traction $\mathbf{n} \cdot \boldsymbol{\sigma}=-i \mathbf{l}(y) \mathrm{e}^{i(k x-\omega t)}$. The $6 \times 6$ matrix of coefficients in (2) is

$\widehat{\mathbf{N}}=-\left(\begin{array}{cc}k(n n)^{-1}(n m) & (n n)^{-1} \\ k^{2}\left[(m n)(n n)^{-1}(n m)-(m m)\right]+\rho \omega^{2} \hat{\mathbf{I}} & k(m n)(n n)^{-1}\end{array}\right)$,

where $(a b)_{j k}=c_{i j k l} a_{i} b_{l}$ with $\mathbf{a}, \mathbf{b} \equiv \mathbf{n}, \mathbf{m}$ and $\hat{\mathbf{I}}$ is the $3 \times 3$ unit matrix. The matrix $\hat{\mathbf{N}}$ has a Hamiltonian structure [37], that is,

$\widehat{\mathbf{N}}=\widehat{\mathbf{T}} \widehat{\mathbf{N}}^{T} \widehat{\mathbf{T}}$,

where $\widehat{\mathbf{T}}$ is a matrix with zero diagonal and unit off-diagonal $3 \times 3$ blocks and the subscript ${ }^{T}$ denotes transposition. We shall disregard dissipation and thus take $\hat{\mathbf{N}}$ to be real.

Solution of Eq. (2) with the initial value $\boldsymbol{\xi}\left(y_{0}\right)$ can be expressed as
$\boldsymbol{\xi}\left(y_{1}\right)=\widehat{\mathbf{M}}\left(y_{1}, y_{0}\right) \boldsymbol{\xi}\left(y_{0}\right)$.

where $\hat{\mathbf{M}}\left(y_{1}, y_{0}\right)$ is the transfer matrix, as it is commonly called in physics. If the medium within $\left[y_{0}, y_{1}\right]$ is a sequence of $j=1, \ldots, n$ homogeneous layers, each characterised by matrix $\widehat{\mathbf{N}}^{(j)}$ (4) with constant $\rho_{j}, \widehat{\mathbf{c}}^{(j)}$ and by thickness $h_{j}$, then

$\widehat{\mathbf{M}}\left(y_{1}, y_{0}\right)=\widehat{\mathbf{M}}_{n} \ldots \widehat{\mathbf{M}}_{2} \widehat{\mathbf{M}}_{1}$ with $\widehat{\mathbf{M}}_{j}=\exp \left(i h_{j} \hat{\mathbf{N}}^{(j)}\right)$.

If $\rho$ and $\widehat{\mathbf{c}}$ vary continuously with $y \in\left[y_{0}, y_{1}\right]$, then $\widehat{\mathbf{M}}\left(y_{1}, y_{0}\right)$ is evaluated by the multiplicative integral of $i \hat{\mathbf{N}}(y)$ [38]. In either case, since $\widehat{\mathbf{N}}$ is Hamiltonian (5) and real, the transfer matrix satisfies the identity $[38,39]$

$\widehat{\mathbf{M}}^{-1}=\widehat{\mathbf{T}} \widehat{\mathbf{M}}^{\dagger} \hat{\mathbf{T}}$,

where the symbol ${ }^{\dagger}$ denotes the Hermitian conjugation.

When a stratified elastic medium is a periodic superlattice, its wave properties are largely characterised by the transfer matrix $\widehat{\mathbf{M}}\left(y_{0}+H, y_{0}\right) \equiv \widehat{\mathbf{M}}$ through a unit cell of thickness (period) $H$ and, more specifically, by its eigenvalues $\gamma_{\alpha}$ and eigenvectors $\zeta_{\alpha} \alpha=1, \ldots, 6$, which depend on $\omega$ and $k$ ( $\zeta_{\alpha}$ also depend on $y_{0}$ ).

By virtue of (8), the eigenvalues $\gamma_{\alpha}$ occur either as complex conjugate pairs $\gamma_{\alpha}=\gamma_{\alpha+3}^{*}$ of unit absolute value $\left|\gamma_{\alpha}\right|=1$ or as pairs $\gamma_{\alpha}=1 / \gamma_{\alpha+3}^{*}$ with $\left|\gamma_{\alpha}\right| \neq 1$ (here and below asterisk implies complex conjugation). These two options, related to each pair $\alpha$ and $\alpha+3$ $(\alpha=1,2,3)$ independently, divide the plane $(\omega, k)$ into alternating domains called, respectively, passbands and stopbands. Their physical meaning is due to the fact that any initial value for Eq. (2) can be expanded as a linear superposition of eigenvectors $\zeta_{\alpha}$, so that the wave solution $\boldsymbol{\xi}(y)$ of (2) considered after passing $m$ unit cells represents the same superposition of eigenvectors but with each $\alpha$-th coefficient multiplied by $\gamma_{\alpha}^{m}$. Aiming at the localised waves, we will be interested in the spectral overlaps of three partial stopbands $\alpha=1,2,3$, i.e., in the "full" stopbands where $\left|\gamma_{\alpha}\right| \neq 1 \forall \alpha$. For brevity, we will refer to them as simply "stopbands" (omitting "full").

Let us fix the numbering of $\gamma_{\alpha}$ in stopbands so that

$\left|\gamma_{\alpha}\right|<1<\left|\gamma_{\alpha+3}\right|, \quad \alpha=1,2,3$,

where $\gamma_{\alpha}=1 / \gamma_{\alpha+3}^{*}$. In view of Eq. (8), the eigenvectors $\zeta_{\alpha}$ inside stopbands satisfy the orthogonality relation

$\boldsymbol{\zeta}_{\alpha}^{\dagger} \hat{\mathbf{T}} \zeta_{\beta}=\delta_{|\alpha-\beta|, 3}, \quad \alpha, \beta=1, \ldots, 6$,

where $\delta_{\alpha, \beta}$ is the Kronecker symbol and the unit normalisation at $\alpha=\beta$ is imposed. The same may be written in the form

$\hat{\mathbf{L}}^{\dagger} \hat{\mathbf{A}}+\hat{\mathbf{A}}^{\dagger} \hat{\mathbf{L}}=\hat{\mathbf{0}}, \quad \hat{\mathbf{L}}^{\prime \dagger} \hat{\mathbf{A}}^{\prime}+\hat{\mathbf{A}}^{\prime \dagger} \hat{\mathbf{L}}^{\prime}=\hat{\mathbf{0}}$

$\hat{\mathbf{L}}^{\prime \dagger} \hat{\mathbf{A}}+\hat{\mathbf{A}}^{\prime \dagger} \hat{\mathbf{L}}=\hat{\mathbf{I}}$

where

$\widehat{\mathbf{A}}=\left(\mathbf{A}_{1}, \mathbf{A}_{2}, \mathbf{A}_{3}\right), \hat{\mathbf{L}}=\left(\mathbf{L}_{1}, \mathbf{L}_{2}, \mathbf{L}_{3}\right)$,

$\widehat{\mathbf{A}}^{\prime}=\left(\mathbf{A}_{4}, \mathbf{A}_{5}, \mathbf{A}_{6}\right), \hat{\mathbf{L}}^{\prime}=\left(\mathbf{L}_{4}, \mathbf{L}_{5}, \mathbf{L}_{6}\right)$

are $3 \times 3$ matrices whose columns $\mathbf{A}_{\alpha}$ and $\mathbf{L}_{\alpha}$ are the first three and second three components of $\zeta_{\alpha}=\left(\mathbf{A}_{\alpha} \mathbf{L}_{\alpha}\right)^{T}$, respectively.

It is at this stage that the case of superlattices with a symmetric unit cell studied in [34] diverges from the present general consideration. By symmetric we mean the unit cells whose arrangement is invariant relative to the midplane. According to [34], the general identity (8) applied to the transfer matrix through a symmetric unit cell splits into two independent identities $\widehat{\mathbf{M}}=\widehat{\mathbf{T}} \widehat{\mathbf{M}}^{T} \widehat{\mathbf{T}}$ and $\widehat{\mathbf{M}}^{-1}=\widehat{\mathbf{M}}^{*}$. This modification does not affect the eigenvalues of $\widehat{\mathbf{M}}$ and hence the band structure, but it provides an additional condition on the eigenvectors, namely, that they can be chosen within the full stopbands as $\zeta_{\alpha}=\zeta_{\alpha+3}^{*}$ which casts relations (10) and (11) in a more determinative form. The latter restrains a scope of options regarding the existence and number of localised waves in superlattices with a symmetric unit cell. 
Correspondingly, a general case of superlattices with asymmetric unit cells considered in the present paper permits a larger variety of possible scenarios for localised waves.

\subsection{Impedance matrices}

Introduce the impedance matrices $\widehat{\mathbf{Z}}$ and $\hat{\mathbf{Z}}^{\prime}$ by means of equivalent definitions

$\mathbf{L}_{\alpha}=-i \widehat{\mathbf{Z}} \mathbf{A}_{\alpha}, \quad \alpha=1,2,3$,

$\mathbf{L}_{\alpha}=i \widehat{\mathbf{Z}}^{\prime} \mathbf{A}_{\alpha}, \quad \alpha=4,5,6$.

or

$\widehat{\mathbf{Z}}=i \widehat{\mathbf{L}} \mathbf{A}^{-1}, \quad \widehat{\mathbf{Z}}^{\prime}=-i \hat{\mathbf{L}}^{\prime} \hat{\mathbf{A}}^{\prime-1}$.

We will be interested in the properties of impedances for $\omega$ and $k$ lying in stopbands. This is where Eqs. (10), (11) hold true and their use together with (14) yields $\widehat{\mathbf{Z}}=\widehat{\mathbf{Z}}^{\dagger}$ and $\widehat{\mathbf{Z}}^{\prime}=\widehat{\mathbf{Z}}^{\prime \dagger}$, i.e. the impedance matrices in stopbands are Hermitian and hence possess real eigenvalues. Note that $\hat{\mathbf{Z}}$ and $\hat{\mathbf{Z}}^{\prime}$ are generally not straightforwardly interrelated, contrary to the case of a symmetric unit cell where $\hat{\mathbf{Z}}^{\prime}=\widehat{\mathbf{Z}}^{T}$. It has been shown [30] that the matrices $\hat{\mathbf{Z}}$ and $\hat{\mathbf{Z}}^{\prime}$ are positive definite at $\omega=0$ and that their frequency derivatives evaluated in stopbands are negative definite. The same is certainly true for the matrix

$\widehat{\mathbf{G}} \equiv \widehat{\mathbf{Z}}+\widehat{\mathbf{Z}}^{\prime}=i\left(\hat{\mathbf{A}} \hat{\mathbf{A}}^{\prime \dagger}\right)^{-1}$,

where the last equality follows from Eqs. $(11)_{2,3}$. In consequence, the eigenvalues of $\widehat{\mathbf{Z}}, \widehat{\mathbf{Z}}^{\prime}$ and $\widehat{\mathbf{G}}$ are positive at $\omega=0$ and they decrease with increasing $\omega$ at fixed $k$.

An essential feature of the matrix $\hat{\mathbf{G}}$ is that none of its eigenvalues can vanish inside stopbands, since

$\widehat{\mathbf{G}}^{-1}=-i \widehat{\mathbf{A}} \hat{\mathbf{A}}^{\prime \dagger} \equiv-i \sum_{\alpha=1}^{3} \mathbf{A}_{\alpha} \otimes \mathbf{A}_{\alpha+3}^{*}$

cannot diverge in there (here $\otimes$ denotes dyadic product). The latter fact is not that obvious because two of the above dyads in $\widehat{\mathrm{G}}^{-1}$ do diverge at the values of $(\omega, k)$ which yield pairwise degeneracy of eigenvalues of the matrix $\widehat{\mathbf{M}}$ and render it non-semisimple (non-diagonalisable). However, it turns out that such discontinuities of the separate dyads in $\widehat{\mathbf{G}}^{-1}$ cancel each other and so $\widehat{\mathbf{G}}^{-1}$ is always finite. This observation was made by using the perturbation theory in [30]. Moreover, in fact a more general formulation of the above property reads that a sum of dyads composed like $\widehat{\mathbf{G}}^{-1}$ from the upper halves of the eigenvectors of $\widehat{\mathbf{M}}$ is always finite for a transfer matrix $\widehat{\mathbf{M}}$ of any size $n \times n$ admitting eigenvalue degeneracy of any multiplicity. The corresponding proof can be based on expressing components of a sum of dyads in question via the eigendecompostions of matrices $\widehat{\mathbf{M}}^{m}$, where $m=0,1, \ldots, n-1$.

The fact that the eigenvalues of $\hat{\mathbf{G}}$ cannot vanish along with their property to decrease with $\omega$ enables further important conclusions, namely, that the eigenvalues $\widehat{\mathbf{G}}$ are positive throughout the lowest stopband and can have at most one pole each in any upper stopband. Hence at most three poles can be shared by six eigenvalues of $\widehat{\mathbf{Z}}, \widehat{\mathbf{Z}}^{\prime}$ in an upper stopband. By definition (14), a pole of eigenvalue of the impedance matrix signifies a surface-wave solution for a half-infinite superlattice with mechanically clamped surface (see [30] for more details).

Let us summarize the main of established properties which will be used below for the analysis of interfacial waves:

eigenvalues of $\hat{\mathbf{Z}}$ and $\hat{\mathbf{Z}}^{\prime}$ in stopbands are

decreasing functions of $\omega$, positive at $\omega=0$;

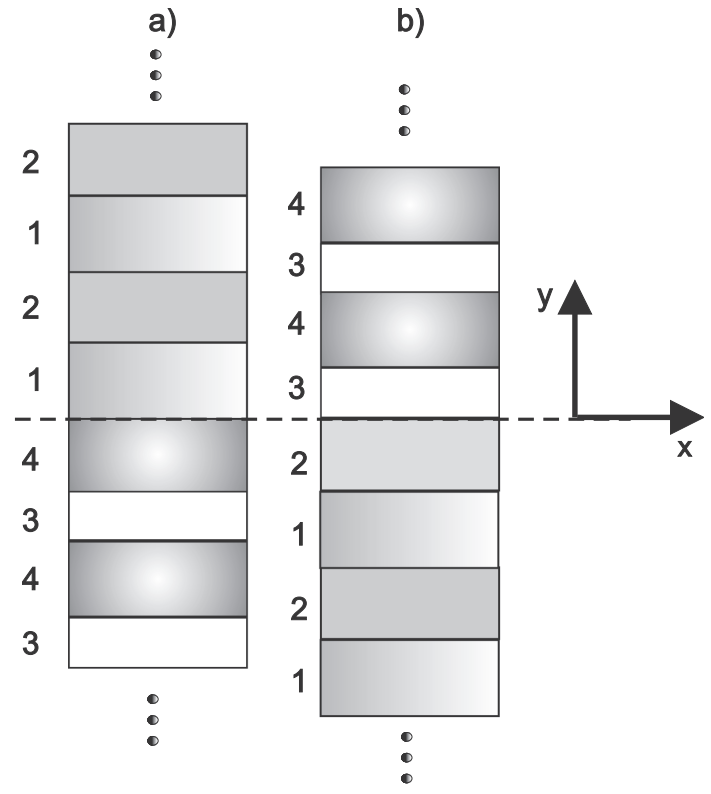

Fig. 1. Example of direct (a) and complementary (b) bicrystals composed of two-layer half-infinite superlattices $J=1$ (layers 1 and 2) and $J=2$ (layers 3 and 4).

eigenvalues of $\widehat{\mathbf{G}}$ in stopbands are non - zero decreasing functions of $\omega$, which are positive in the lowest stopband and admit at most one pole each in an upper stopbands at fixed $k$.

\section{Interfacial waves}

\subsection{Unrestricted anisotropy}

\subsubsection{Dispersion equation}

Consider a 1D bicrystal consisting of two perfectly bonded half-infinite periodic superlattices. Each, or at least one of them, is supposed to have an asymmetric unit cell (otherwise see [34]). Let the bonding interface be the plane $y=0$ and the superlattices occupying the halfspaces $y \geqslant 0$ and $y \leqslant 0$ be labeled by indices $J=1$ and $J=2$, respectively. Alongside a given bicrystal, we will consider its counterpart consisting of the superlattice $J=2$ at $y \geqslant 0$ and of the superlattice $J=1$ at $y \leqslant 0$. These two mutually swapped structures are referred to in the following as "direct" and "complementary" bicrystals, respectively (see an example in Fig. 1).

According to Eq. (6), spatial evolution of the displacement-traction field at the successive layer interfaces, which are counted away from the plane $y=0$ into the depths of adjoined superlattices with the periods $H_{1}(y \geqslant 0)$ and $H_{2}(y \leqslant 0)$, is defined by the transfer matrices $\widehat{\mathbf{M}}^{(1)}\left(H_{1}, 0\right) \equiv \widehat{\mathbf{M}}^{(1)}$ and $\hat{\mathbf{M}}^{(2)}\left(-H_{2}, 0\right)=\widehat{\mathbf{M}}^{(2)}\left(0, H_{2}\right)=\left[\widehat{\mathbf{M}}^{(2)}\left(H_{2}, 0\right)\right]^{-1}$ $\equiv \widehat{\mathbf{M}}^{(2)-1}$ in the direct bicrystal and by $\widehat{\mathbf{M}}^{(2)}$ and $\widehat{\mathbf{M}}^{(1)-1}$ in the complementary one. Recall that a matrix and its inverse have the same eigenvectors and mutually inverse sets of eigenvalues, hence the direct and complementary bicrystals imply identically the same partitioning of the $(\omega, k)$-plane into the pass bands and stopbands defined with respect to the eigenvalue spectra of matrices $\widehat{\mathbf{M}}^{(1)}$ and $\widehat{\mathbf{M}}^{(2)}$. We are interested in the interfacial acoustic waves (IAWs) localised at the interface $y=0$ and vanishing at the infinite distance $y \rightarrow \pm \infty$. Such waves should typically exist in the overlaps of the "full" stopbands of both constituent superlattices $J=1$ and $J=2$, i.e. in the ranges of $(\omega, k)$ values where none of the eigenvalues $\gamma_{\alpha}^{(1)}$ and $\gamma_{\alpha}^{(2)}$ of $\widehat{\mathbf{M}}^{(1)}$ and $\widehat{\mathbf{M}}^{(2)}$ has unit absolute value. So-defined overlaps will be called the stopbands of a bicrystal and denoted by $0<\omega<\omega_{u}$ (the lowest stopband) and $\omega_{l}<\omega<\omega_{u}$ (upper stopbands), where the lower and upper band 
edges $\omega_{l}$ and $\omega_{u}$ are functions of $k$.

With regard for the numbering convention (9) applied to the eigenvalues $\gamma_{\alpha}^{(J)}$ of $\widehat{\mathbf{M}}^{(J)}(J=1,2)$, the IAW solutions $\boldsymbol{\xi}^{(J)}(y)$ of Eq. (2) in the superlattices $J=1$ at $y \geqslant 0$ and $J=2$ at $y \leqslant 0$ of the direct bicrystal should be generated by the initial values $\xi^{(1)}(0)=\sum_{\alpha=1}^{3} c_{\alpha} \zeta_{\alpha}^{(1)}$ and $\xi^{(2)}(0)=\sum_{\alpha=4}^{6} d_{\alpha} \zeta_{\alpha}^{(2)}$ at $y=0$. The continuity of the displacement and traction requires that

$\sum_{\alpha=1}^{2} c_{\alpha} \zeta_{\alpha}^{(1)}=\sum_{\alpha=3}^{4} d_{\alpha} \zeta_{\alpha}^{(2)}$,

or, equivalently,

$\mathbf{U}=\mathbf{U}^{\prime}, \quad \widehat{\mathbf{Z}}^{(1)} \mathbf{U}=-\widehat{\mathbf{Z}}^{(2)} \mathbf{U}^{\prime}$,

where $\mathbf{U}=\sum_{\alpha=1}^{3} c_{\alpha} \mathbf{U}_{\alpha}^{(1)}, \mathbf{U}^{\prime}=\sum_{\alpha=4}^{6} d_{\alpha} \mathbf{U}_{\alpha}^{(2)}$, and $\widehat{\mathbf{Z}}^{(1)}, \widehat{\mathbf{z}}^{\prime(2)}$ are the impedance matrices defined by Eq. (13) for the superlattices $J=1$ and 2, respectively. Hence, the dispersion equation for IAWs in the direct bicrystal is

$\operatorname{det} \widehat{\mathbf{Z}}_{B}=0$ with $\widehat{\mathbf{Z}}_{B}=\widehat{\mathbf{Z}}^{(1)}+\widehat{\mathbf{Z}}^{(2)}$,

where the subscript $B$ means "bicrystal" (instead of the notation $\widehat{\mathbf{Z}}_{I}$ with $I$ for "interface" as used in [34]). Swapping labels $J=1$ and 2 in (21) yields the dispersion equation for IAWs in the complementary bicrystal,

$\operatorname{det} \widehat{\mathbf{Z}}_{B}^{\prime}=0$ with $\widehat{\mathbf{Z}}_{B}^{\prime}=\widehat{\mathbf{Z}}^{(2)}+\widehat{\mathbf{Z}}^{(1)}$.

It is evident that (21) and (22) are quite different equations and so the IAWs in the same stopbands of the direct and complementary bicrystals do not at all coincide; for instance, a given stopband may well admit an IAW for the direct bicrystal and does not admit it for the complementary one, or vice versa. However, we will demonstrate that the IAW existence in these two bicrystal structures is particularly correlated. With this in mind, introduce the matrix

$$
\widehat{\mathbf{G}}_{B}=\widehat{\mathbf{Z}}_{B}+\widehat{\mathbf{Z}}_{B}^{\prime}=\widehat{\mathbf{G}}_{1}+\widehat{\mathbf{G}}_{2}
$$

with $\widehat{\mathbf{G}}_{J}=\widehat{\mathbf{Z}}^{(J)}+\widehat{\mathbf{Z}}^{(J)^{\prime}}, \quad J=1,2$.

For the future use, note the identity

$\widehat{\mathbf{G}}_{B}=\sum_{\alpha=1}^{3}\left(\lambda_{\alpha} \mathbf{e}_{\alpha} \mathbf{e}_{\alpha}^{\dagger}+\lambda_{\alpha}^{\prime} \mathbf{e}_{\alpha}^{\prime} \mathbf{e}_{\alpha}^{\prime}\right)$

$=\sum_{\alpha=1}^{3}\left(g_{\alpha}^{(1)} \mathbf{f}_{\alpha}^{(1)} \mathbf{f}_{\alpha}^{(1) \dagger}+g_{\alpha}^{(2)} \mathbf{f}_{\alpha}^{(2)} \mathbf{f}_{\alpha}^{(2) \dagger}\right)$,

where $\lambda_{\alpha}, \mathbf{e}_{\alpha}$ and $\lambda_{\alpha}^{\prime}, \mathbf{e}_{\alpha}^{\prime}$ are the eigenvalues and eigenvectors of $\widehat{\mathbf{Z}}_{B}$ and $\widehat{\mathbf{Z}}_{B}^{\prime}$, respectively; $g_{\alpha}^{(J)}$ and $\mathbf{f}_{\alpha}^{(J)}$ are the eigenvalues and eigenvectors of $\widehat{\mathbf{G}}_{J}$ $(\alpha=1,2,3 ; J=1,2)$.

\subsubsection{IAWs in the lowest stopband}

As follows from (17) and the definition of $\widehat{\mathbf{Z}}_{B}$ and $\widehat{\mathbf{Z}}_{B}^{\prime}$, each of their eigenvalues is positive at $\omega=0$, decrease with growing $\omega$ and has no poles within the lowest stopband $0<\omega<\omega_{u}$. Since their zeros are the solutions of the dispersion Eqs. (21) and (22), it follows that the direct and complementary bicrystals admit the existence of at most three IAWs each in the lowest stopband at any fixed $k$. However, a stronger statement can be proved if we consider these two bicrystal structures simultaneously and invoke the matrix $\widehat{\mathbf{G}}_{B}$. By (18) and (23), $\widehat{\mathbf{G}}_{B}$ is positive definite throughout the lowest stopband and, in particular, at its upper edge $\omega_{u}$. Hence no more than three out of six eigenvalues $\lambda_{\alpha}, \lambda_{\alpha}^{\prime}$, of $\widehat{\mathbf{Z}}_{B}$ and $\widehat{\mathbf{Z}}_{B}^{\prime}$ can be negative at $\omega_{u}$. Indeed, assume that there are four negative and only two positive ones, say, $\lambda_{1,2}, \lambda_{1,2}^{\prime}<0$ and $\lambda_{3}, \lambda_{3}^{\prime}>0$. Then multiplying the first equality in (24) from the right and left sides by the vector product $\mathbf{e}_{3} \times \mathbf{e}_{3}^{\prime} \equiv \mathbf{p}$ and its complex conjugate $\mathbf{p}^{*}$ yields

$\mathbf{p}^{\dagger} \hat{\mathbf{G}}_{B} \mathbf{p}=\sum_{\alpha=1}^{2}\left(\lambda_{\alpha}\left|\mathbf{p}^{\dagger} \mathbf{e}_{\alpha}\right|^{2}+\lambda_{\alpha}^{\prime}\left|\mathbf{p}^{\dagger} \mathbf{e}_{\alpha}^{\prime}\right|\right)<0$ at $\omega_{u}$, which contradicts positive definiteness of $\widehat{\mathbf{G}}_{B}$. Thus, at any fixed $k$ and $\omega$ in the lowest stopband, the number of zeros of eigenvalues of $\widehat{\mathbf{Z}}_{B}$ and $\widehat{\mathbf{Z}}_{B}^{\prime}$, i.e. the total number of solutions of Eqs. (21) and (22), cannot exceed three. We can therefore conclude that

given a fixed $k$, at most three IAWs can exist in the lowest stopband for the direct and complementary bicrystals in total.

It is clear that this result is in no conflict with the possibility of up to three IAWs in the direct bicrystal or in the complementary one; at the same time, (26) is a stronger statement in the sense that it additionally restricts the total number of IAWs in these structures. For instance, it tells us that if two IAWs occur in the lowest stopband for a given bicrystal then at most one IAWs can exist within this band for its swapped counterpart and vice versa. Note to this end that only one IAW at fixed $k$ is allowed in the lowest stopband of a bicrystal with symmetric unit cells of both constituent superlattices [34].

\subsubsection{IAWs in upper stopbands}

By (17), any eigenvalue of matrices $\widehat{\mathbf{Z}}_{B}$ and $\hat{\mathbf{Z}}_{B}^{\prime}$ may have at most two zeros (one before and one after the pole) in an upper stopband $\omega_{l}<\omega<\omega_{u}$ at fixed $k$, so that each of Eqs. (21) and (22) may have at most six solutions, i.e. a direct and complementary bicrystals admit at most six IAWs each. Note that this is twice the maximum number of IAWs which are possible for a bicrystal with symmetric unit cells [34]. Now, like in the case of the lowest stopband, we will show that the existence of IAWs in the direct and complementary bicrystals is correlated in the sense that the maximum number of six IAWs at fixed $k$ per upper stopband in each bicrystal is also the supremum for the aggregate number of IAWs for both bicrystals within a given stopband.

If none of six eigenvalues of $\widehat{\mathbf{Z}}_{B}$ and $\widehat{\mathbf{Z}}_{B}^{\prime}$ has a pole in a stopband at given $k$, i.e. they all are monotone in $\omega$, then the total number of eigenvalue zeros for $\widehat{\mathbf{Z}}_{B}$ and $\widehat{\mathbf{Z}}_{B}^{\prime}$ cannot exceed six. Let us pose a question whether an occurrence of eigenvalue pole(s) for $\widehat{\mathbf{Z}}_{B}$ and/or $\widehat{\mathbf{Z}}_{B}^{\prime}$ (and hence for $\widehat{\mathbf{G}}_{1}$ and/or $\widehat{\mathbf{G}}_{2}$ ) may augment this upper bound, i.e. may permit Eqs. (21) and (22) to have more than six roots in total and so more than six IAWs to be shared by the direct and supplementary bicrystals in the same upper stopband and fixed $k$.

Suppose first that there is one eigenvalue pole inside a given stopband. In this case, in order for the number of zeros of eigenvalues $\lambda_{\alpha}, \lambda_{\alpha}^{\prime}$ ( $\alpha=1,2,3)$ of $\widehat{\mathbf{Z}}_{B}$ and $\hat{\mathbf{Z}}_{B}^{\prime}$ to exceed six, all six of them must be positive at the lower stopband edge $\omega_{l}$ and negative at the upper band edge $\omega_{u}$, so that both $\widehat{\mathbf{Z}}_{B}$ and $\widehat{\mathbf{Z}}_{B}^{\prime}$ and hence $\widehat{\mathbf{G}}_{B}$ must be positive definite at $\omega_{l}$ and negative definite at $\omega_{u}$. Under this condition, manipulating the second equality in (24) similarly to (25) demonstrates that at least three among six eigenvalues $g_{\alpha}^{(J)}$ of $\widehat{\mathbf{G}}_{J}(J=1,2)$ must be positive at $\omega_{l}$ and at least three be negative at $\omega_{u}$. At the same time, by (18), none of them can vanish in a stopband, so the eigenvalue with a pole must be negative at $\omega_{l}$ and positive at $\omega_{u}$, whereas the continuous ones must be either all positive or all negative throughout the given stopband. Hence, given one eigenvalue pole, the above requirement of at least three positive $g_{\alpha}^{(J)}$ at $\omega_{l}$ implies that at least four $g_{\alpha}^{(J)}$ must be positive at $\omega_{u}$, while at least three negative $g_{\alpha}^{(J)}$ at $\omega_{u}$ entail at least four $g_{\alpha}^{(J)}$ are negative at $\omega_{l}$. For example, let the latter four ones be $g_{\alpha}^{(J)}, \alpha=1,2 ; J=1$, 2. Then multiplying the second equality in (24) from the right and left sides by the vectors $\mathbf{t}_{3}^{(1)} \times \mathbf{t}_{3}^{(2)} \equiv \mathbf{q}$ and $\mathbf{q}^{*}$ yields

$\mathbf{q}^{\dagger} \widehat{\mathbf{G}}_{B} \mathbf{q}=\sum_{\alpha=1}^{2}\left(g_{\alpha}^{(1)}\left|\mathbf{q}_{\alpha}^{\dagger} \mathbf{t}_{\alpha}^{(1)}\right|^{2}+g_{\alpha}^{(2)}\left|\mathbf{q}^{\dagger} \mathbf{t}_{\alpha}^{(2)}\right|^{2}\right)<0$ at $\omega_{l}$,

which contradicts positive definiteness of $\widehat{\mathbf{G}}_{B}$ at $\omega_{l}$. Similarly, occurrence of at least four positive $g_{\alpha}^{(J)}$ at $\omega_{u}$ rules out negative negativeness $\widehat{\mathbf{G}}_{B}$. Thus the occasion of a single eigenvalue pole of $\widehat{\mathbf{Z}}_{B}$ and $\widehat{\mathbf{Z}}_{B}^{\prime}$ does not raise the upper bound of six for the possible number of IAWs per an upper stopband and fixed $k$ in direct and supplementary bicrystals in total. 
Next let us assume that two eigenvalue poles occur in a given stopband. Now the questioned possibility of more than six zeros of eigenvalues $\lambda_{\alpha}$, $\lambda_{\alpha}^{\prime}$ of $\widehat{\mathbf{Z}}_{B}$ and $\widehat{\mathbf{Z}}_{B}^{\prime}$ necessitates one of the following options:

(i) all six $\lambda_{\alpha}, \lambda_{\alpha}^{\prime}(\alpha=1,2,3)$ are positive at $\omega_{l}$ and at most one is positive at $\omega_{u}$

(ii) one of $\lambda_{\alpha}, \lambda_{\alpha}^{\prime}$ is negative at $\omega_{l}$ and all six are negative at $\omega_{u}$, so that $\widehat{\mathbf{G}}_{B}$ cannot be negative definite at $\omega_{l}$ and it is such at $\omega_{u}$.

At the same time, by assumption, there are two discontinuous and four monotone branches of eigenvalues $g_{\alpha}^{(J)}(\omega)$ of of $\widehat{\mathbf{G}}_{J}(J=1$, 2), i.e. at least two of $g_{\alpha}^{(J)}$ are negative at $\omega_{l}$ and at least two of $g_{\alpha}^{(J)}$ are positive at $\omega_{u}$. Assume the case (i), so that $\widehat{\mathbf{G}}_{B}$ is positive definite at $\omega_{l}$ and it cannot have this property at $\omega_{u}$. Then, by analogy with (27), there must be no less than either four or three positive eigenvalues $g_{\alpha}^{(J)}$ at $\omega_{l}$ which entail either six or five positive $g_{\alpha}^{(J)}$ at $\omega_{u}$, respectively. The former implies positive definiteness of $\widehat{\mathbf{G}}_{B}$ at $\omega_{u}$ and hence contradicts the case (i). The latter means that, at $\omega_{u}$, one positive eigenvalue of $\widehat{\mathbf{z}}_{B}$ and $\widehat{\mathbf{z}}_{B}^{\prime}$, say $\lambda_{1}$, coexists with one negative eigenvalue of $\widehat{\mathbf{G}}_{J}$ or $\widehat{\mathbf{G}}_{2}$, say $g_{1}^{(1)}$. Multiplying two equalities in (24) from the right and left sides by the vectors $\mathbf{t}_{1}^{(1)} \times \mathbf{e}_{1} \equiv \mathbf{s}$ and $\mathbf{s}^{*}$ leads to incompatible inequalities

$\mathbf{s}^{*} \widehat{\mathbf{G}}_{B} \mathbf{S}=\sum_{\alpha=2}^{3} \lambda_{\alpha}\left|\mathbf{s}^{\dagger} \mathbf{e}_{\alpha}\right|^{2}+\sum_{\alpha=1}^{3} \lambda_{\alpha}^{\prime}\left|\mathbf{s}^{\dagger} \mathbf{e}_{\alpha}^{\prime}\right|^{2}<0$,

$\mathbf{s}^{*} \widehat{\mathbf{G}}_{B} \mathbf{S}=\sum_{\alpha=2}^{3} g_{\alpha}^{(1)}\left|\mathbf{s} \mathbf{f}_{\alpha}^{(1)}\right|^{2}+\sum_{\alpha=1}^{3} g_{\alpha}^{(2)}\left|\mathbf{s} \mathbf{f}_{\alpha}^{(2)}\right|^{2}>0$.

Thus option (i) is inconsistent and should be rejected. In turn, option (ii) implies that $\widehat{\mathbf{G}}_{B}$ is negative definite at $\omega_{u}$ and hence, in the presence of two poles, four or three eigenvalues of $\widehat{\mathbf{G}}_{1}$ and $\widehat{\mathbf{G}}_{2}$ are negative at $\omega_{u}$ and six or five ones are negative at $\omega_{l}$. By analogous reasoning, option (ii) can also be ruled out.

Considerations for the cases of more than two (up to six) eigenvalue poles in a stopband are further ramified but similar in the essence. It is straightforward to verify that none of these occasions can yield more than six eigenvalue zeros for $\widehat{\mathbf{Z}}_{B}$ and $\widehat{\mathbf{Z}}_{B}^{\prime}$. Thus we can conclude that

given a fixed $k$, at most six IAWs can exist in an upper stopband for the direct and complementary bicrystals in total.

\subsection{Monoclinic symmetry}

All the preceding considerations hold true under no assumptions regarding the crystallographic symmetry and orientation of the involved materials. Now we consider the case where all constituent layers of a bicrystal are of at least monoclinic symmetry and their stiffness tensors possess a common symmetry plane orthogonal to the layer interfaces. The wave Eq. (2) in the sagittal plane $X Y$ coinciding with the symmetry plane splits into two equations for the modes of shear horisontal and sagittal polarizations ( $\mathrm{SH}$ - and S-modes, respectively). So do the transfer and impedance matrices. Correspondingly, the interfacial waves consisting of $\mathrm{SH}$ - and of S-modes are uncoupled from each other and may therefore be considered separately. Note that the stopbands where these waves occur are also mutually independent.

\subsubsection{SH-IAWs}

The dispersion equations defining SH-IAWs within stopbands in the direct and complementary bicrystals, respectively, are the uncoupled zz-components of Eqs. (21) and (22), namely,

$Z_{B, z z}=0$ with $Z_{B, z z}=Z_{z z}^{(1)}+Z_{z z}^{\prime(2)}$,

$Z_{B, z z}^{\prime}=0$ with $Z_{B, z z}^{\prime}=Z_{z z}^{(2)}+Z_{z z}^{\prime(1)}$. of impedances described in Section 2.1 and also fulfil some particular features following explicitly due to the scalar format. In summary, $Z_{S H}^{(J)}(\omega)$ and $Z_{S H}^{\prime(J)}(\omega)$ taken at $k$ fixed and $\omega$ varying in stopbands are real decreasing functions, positive at $\omega=0$; in the lowest stopband $0<\omega<\omega_{u}$, they are continuous and one of $Z_{S H}^{(J)}(\omega)$ and $Z_{S H}^{\prime(J)}(\omega)$ related to the same superlattice (same $J$ ) stays positive while the other has one zero; in an upper stopband $\omega_{l}<\omega<\omega_{u}$, the functions $Z_{S H}^{(J)}(\omega)$ and $Z_{S H}^{\prime(J)}(\omega)$ with the same $J$ have one zero and one pole, which either both occur for one or the other function or occur by one for different functions. Besides, they satisfy

$Z_{z z}^{(J)}+Z_{z z}^{\prime(J)}=0$ at $\omega=\omega_{l}(\neq 0)$ and

at $\omega=\omega_{u} \quad(J=1$ or 2$)$.

In particular, bearing in mind that the SH impedance decrease with $\omega$, it follows from (33) that the impedance value at the lower edge $\omega_{l}$ is greater (respectively, less) than its value at the upper edge $\omega_{u}$ at the same $k$ if this impedance is continuous (respectively, has a pole) within this stopband. For more details, see [40].

Consider SH-IAWs in the lowest stopband $0<\omega<\omega_{u}$. It follows from the above that each of the aggregate functions $Z_{B, z z}$ and $Z_{B, z z}^{\prime}$ on the left-hand sides (1.h.s.) of Eqs. (31) and (32) may have one and only one zero, i.e., no more than one SH-IAW can exist in the lowest stopband for any given (direct) bicrystal and the same is true for the complementary bicrystal. This being stated, an additional argument rules out the possibility of these SH-IAWs to emerge by one for both direct and complementary bicrystals. Indeed, the latter would imply that both $Z_{B, z z}<0$ and $Z_{B, z z}^{\prime}<0$ at $\omega_{u}$, i.e.

$Z_{B, z z}+Z_{B, z z}^{\prime}=Z_{z z}^{(1)}+Z_{z z}^{\prime(2)}+Z_{z z}^{(2)}+Z_{z z}^{\prime(1)}<0$

at $\omega=\omega_{u}$

which is impossible due to (33). Thus the above statement can be sharpened to the form:

the lowest stopband of a bicrystal admits at most one SH - IAW per fixed $k$ which occurs alternatively

in either direct or complementary bicrystal.

Consider SH-IAWs in an upper stopband $\omega_{l}<\omega<\omega_{u}$ ( $k$ being fixed). Note that the 1.h.s. function of any one of Eqs. (31) and (32), say $Z_{B, z z}(\omega)$ in (31), has two zeros either if $Z_{B, z z}$ has one pole and is positive at $\omega_{l}$ and negative at $\omega_{u}$ or if $Z_{B, z z}$ has two poles, is of the same sign at both band edges and its value at $\omega_{l}$ is less than at $\omega_{u}$. In the former case, by (33), the l.h.s. function $Z_{B, z z}^{\prime}(\omega)$ in Eq. (32) is negative at $\omega_{l}$ and positive at $\omega_{u}$, hence, being a decreasing function, it must have a pole but no zeros. In the latter case, the function $Z_{B, z Z}^{\prime}(\omega)$ is continuous and, by (33), is sign-definite throughout the stopband. Thus in any case if one of Eqs. (31) has two solutions, then the other has none. Thus we arrive at the following conclusion:

given a fixed $k$, at most two $\mathrm{SH}$ - IAWs can exist in an upper stopband for the direct and complementary bicrystals in total.

\subsubsection{S-IAWs}

The dispersion equations defining S-IAWs have the same form as Eqs. (21) and (22) but applied to the S-impedances which are the $2 \times 2$ upper diagonal blocks of the $3 \times 3$ impedances $\widehat{\mathbf{Z}}^{(J)}$ and $\widehat{\mathbf{Z}}^{(J)}$. The fact that S-impedances are matrices, not scalars like SH-ones, does not allow the conclusions as detailed as in the $\mathrm{SH}$ case. Adapting the considerations presented in Section 3.1 to the case of $2 \times 2$ matrices leads to the following statements:

given a fixed $k$, at most two S - IAWs can exist in the lowest stop

band for the direct and complementary bicrystals in total; 
given a fixed $k$, at most four S - IAWs can exist in an upper stop

band for the direct and complementary bicrystals in total.

\section{Maximum number of IAWs: Examples}

It was found above that the number of IAWs per stopband cannot be greater than the certain quantity. The purpose of the present section is to prove by evidence that this upper bound is really attainable (i.e., in formal terms, that this bound is a maximum rather than supremum). With this in mind, we present below a number of examples where the maximum possible number of IAWs does occur in a canonical bilayered structure involving the materials of practical use and the model ones.

Consider an infinite sequence of alternating layers of materials $\mathrm{A}$ and $B$ which all have the same thickness $h$ except a single layer, say of material A, with the thickness $d$ being less than $2 h$. Such structure implying a multilayered composite with a period $H=2 h$ perturbed by an embedded structural defect is of its own interest and it is also suitable for numerical modeling. The structure at hand emulates a bicrystal (composite superlattice) whose interface $y=0$ is a fictitious plane dividing the defect layer into two layers $y \in\left[0, h_{1}\right]$ and $y \in\left[-h_{2}, 0\right]$ where $h_{1}+h_{2}=d$. Correspondingly, the upper halfspace $y \geqslant 0$ may be seen as a superlattice with a unit cell comprising A/B/A layers of successive thicknesses $\left\{h_{1}, h, h-h_{1}\right\}$, while the lower halfspace $y \leqslant 0$ is a superlattice with an $\mathrm{A} / \mathrm{B} / \mathrm{A}$ unit cell of thicknesses $\left\{h-h_{2}, h, h_{2}\right\}$ (both triplets of layer thicknesses are ordered in the positive $y$-direction). Referring to this structure as a direct bicrystal, we mentally swap its two halves to arrive at its complementary counterpart as defined in Section 3.1.1. By construction, this complementary bicrystal is also an infinite sequence of alternating equidistant $\mathrm{A}$ and $\mathrm{B}$ layers with a period $H=2 h$ perturbed by an embedded defect A layer, whose thickness is $d^{\prime}=H-d$. Both above models of direct and complementary bicrystals are shown in Fig. 2.

Note aside that the location of the fictitious interface inside the defect layer of a given a thickness $d$ (i.e. splitting $d$ into $h_{1}$ and $h_{2}$ ) makes no impact on the IAW dispersion spectrum. This is physically evident indeed, since the wave localisation and guidance are due to the defect layer as a whole. From the formal perspective of a bicrystal

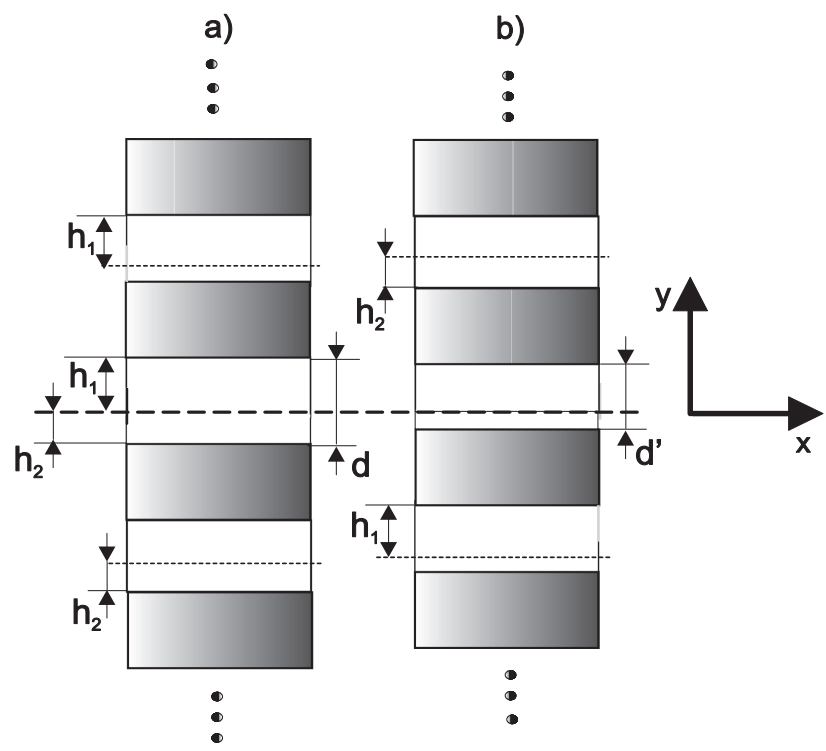

Fig. 2. Direct (a) and complementary (b) bicrystals composed of layers of materials A and B (white and grey domains respectively). Dashed line shows a fictitious interface $y=0$ cutting the defect A layer. Dotted lines mark the edges of A/B/A unit cells. Thicknesses of layers constituting the unit cells are indicated at left. model, this follows from the fact that shifting the period edge from the point $y=0$ to $y=\Delta y$ changes each of the transfer matrices $\widehat{\mathbf{M}}^{(J)}(H, 0) \equiv \widehat{\mathbf{M}}^{(J)}, J=1,2$, for the constitutive halves of the bicrystal to its similar matrix

$\widehat{\mathbf{M}}^{(J)}(H+\Delta y, \Delta y)=\widehat{\mathbf{M}}_{\Delta} \widehat{\mathbf{M}}^{(J)} \widehat{\mathbf{M}}_{\Delta}^{-1}$,

where $\widehat{\mathbf{M}}_{\Delta} \equiv \widehat{\mathbf{M}}(\Delta y, 0)$ is the transfer matrix for the defect layer material. Hence the "shifted" transfer matrices have the same eigenvalues $\gamma_{\alpha}$ as either of $\widehat{\mathbf{M}}^{(J)}$ (which are also similar to each other for the given model). Their eigenvectors are obtained by multiplying all eigenvectors $\zeta_{\alpha}^{(J)}$ of $\widehat{\mathbf{M}}^{(J)}$ by the same matrix $\widehat{\mathbf{M}}_{\Delta}^{-1}$; this confirms the invariance of the band structure and of the roots of the boundary Eq. (19) with respect to $\Delta y$.

For the first set of illustrations, assume the A and B materials to be epitaxially grown GaAs and AlAs [41] with density $\rho=5360$ and $\rho=3760 \quad$ (in $\mathrm{kg} / \mathrm{m}^{3}$ ) and stiffness coefficients $c_{11}=118.8, c_{12}=53.8, c_{44}=59.4$ and $c_{11}=120.2, c_{12}=57, c_{44}=58.9$ (in GPa units), respectively [42]. Let the defect layer be of GaAs and its thickness be $d=1.1 \mathrm{~h}$ in the direct bicrystal and hence $d^{\prime}=0.9 \mathrm{~h}$ in the complementary one. All the layers are of cubic symmetry and have an identical orientation. The plane of layer interfaces and the sagittal plane are orthogonal to the principal (i.e., fourfold) symmetry axes, hence the sought IAWs split into S- and SH-polarized ones.

Basing on the above material data, examples of the S-IAW and SHIAW dispersion branches are calculated and displayed in Figs. 3 and $4 \mathrm{a}$. Fig. 3 displays the IAW branches in the lowest SH stopband (which is the area below the dark grey strip indicating the first $\mathrm{SH}$ passband) and in one of the upper S stopbands (the blank strip). Note that the latter is the third stopband, so that there are two more S stopbands alternating with $\mathrm{S}$ passbands within the lower light grey area and that there is a second SH stopband within the upper light grey area (the wave branches in the light grey spectral ranges in this and subsequent figures are beyond our attention).

It is seen from Fig. 3 that the lowest SH stopband contains a single SH-IAW branch, which comes about in the direct bicrystal whereas no such wave exists for the complementary bicrystal. This is in accordance with statement (35). In turn, the upper S stopband contains four S-IAW branches, which come about pairwise in the direct and complementary bicrystals. This observation exemplifies the occurrence of the maximum number of four S-IAW branches per stopband which was mentioned in (38).

Fig. 4a demonstrates two S-IAW branches in the lowest S stopband which both occur in the direct bicrystal. By (37), two is the maximum possible total number of S-IAWs in a lowest stopband. Correspondingly, there must be no S-IAWs in the complementary bicrystal and the computations confirm this fact.

Let us now consider IAWs of general polarisation and confirm that the maximum number of such waves at $k$ fixed, which is established by (26) and (30) as three in the lowest stopband and six in an upper stopband, is a possible event. This can readily be visualised via the following model examples. The idea of the first example is to modify the material constants so that the spectral areas occupied by the lowest $\mathrm{SH}$ stopband and the lowest $S$ stopband would be almost congruent and their overlap would contain the SH-IAW branch along with two S-IAW branches, which is the maximum possible number of IAWs of both polarisations. This state of affairs is realised in the structure of "modified" GaAs and AlAs layers whose actual shear moduli $c_{44}=c_{55}$ are replaced with the values 50 and $50.9 \mathrm{GPa}$, respectively, in order to appropriately adjust parameters of SH modes. Resulting overlap of SH and $\mathrm{S}$ lowest stopbands which contains one SH-IAW and two S-IAW branches is presented in Fig. $4 \mathrm{~b}$; moreover, all these three wave branches correspond to the direct bicrystal (hence the complementary bicrystal does not admit any SH- and S-IAWs with frequency in the lowest stopband). The constructed example with $\mathrm{SH}$ and $\mathrm{S}$ waves in fact suffices to infer the desired conclusion on waves of general polarisation. 

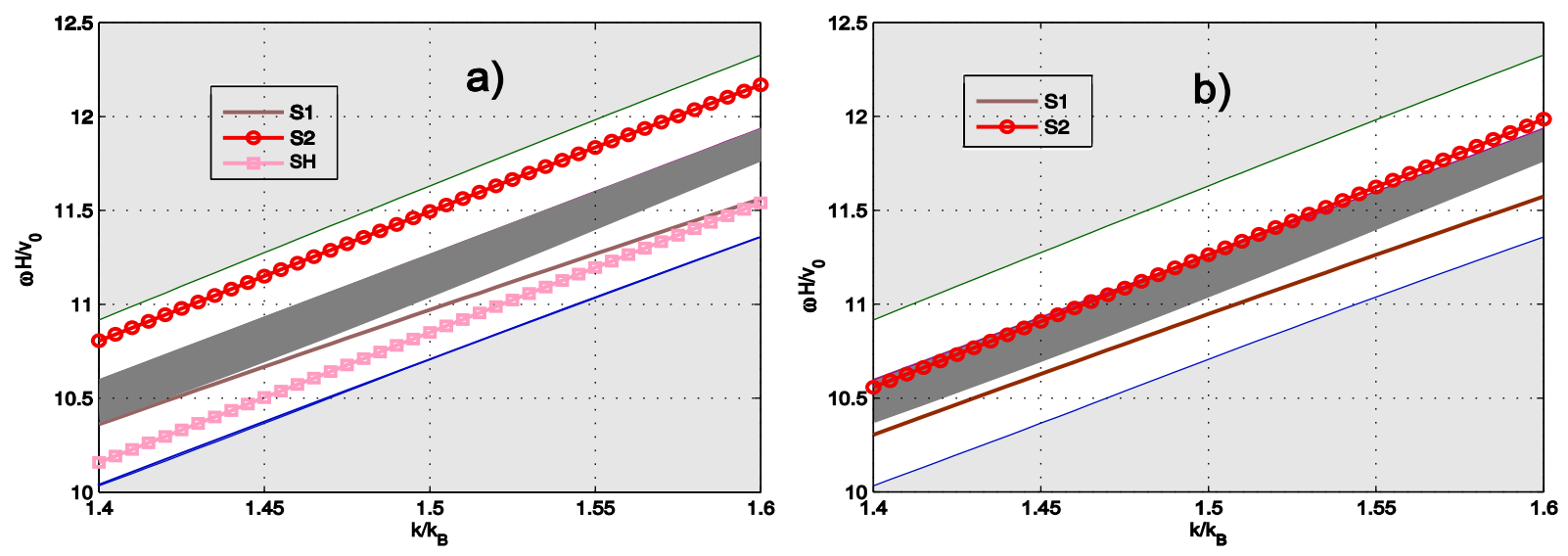

Fig. 3. S-IAW and SH-IAW dispersion branches in the direct (a) and complementary (b) bicrystals composed of the GaAs and AlAs layers. Notation of the branches is explained in the insets. Dark grey strip is the first SH passband, so that the area below is the lowest SH stopband. Blank strip is an upper (the third) S stopband. $k_{B}=2 \pi / H$ and $v_{0}=3000 \mathrm{~m} / \mathrm{s}$.

Indeed, it is clear that a small enough disturbance of the geometrical setup, i.e. deviation of the axes $X$ and/or $Y$ from their initial orientation parallel to the principal symmetry axes, should retain the above three dispersion branches in the lowest stopband which correspond, due to the SH/S mode mixing, to waves of general polarisation.

As an example, Fig. 5 shows the frequencies of IAWs, which exist at $k=1.55 k_{B}$ in the lowest stopband of the direct bicrystal (see Fig. 2a), as functions of the thickness $d$ of the defect layer. The direction of propagation $X$ makes an angle $\varphi=3^{\circ}$ with a fourfold symmetry axis of "modified" GaAs and AlAs layers, while the axis $Y$ remains parallel to another fourfold symmetry axis. The three waves displayed in Fig. 5 originate from two S-IAWs and one SH-IAW existing at $\varphi=0^{\circ}$ (see Fig. 4a); correspondingly, two of them at $\varphi=3^{\circ}$ are quasiS-IAWs (curves 1 and 2) and one is quasiSH-IAW (curve 3), where "quasi" implies that the polarizations are close to a pure sagittal or pure shearhorizontal one. To be more precise, the curves 2 and 3 correspond to quasiS-IAWs and quasiSH-IAWs, respectively, for $d / h>1.06$, but then curves 2 and 3 come very near to each other at $d / h=1.06$ and the hybridization effect swaps their polarization type, so that the curves 2 and 3 become quasiSH and quasiS, respectively, for $d / h<1.06$. Simultaneously with that, curve 1 at $d / h=1.06$ reaches the stopband edge and thereby terminates, i.e., the corresponding quasiS IAW ceases to exist as a localized wave. For $d / h>1.06$, this S-IAW consists of two partial quasi-S modes with a small admixture of quasi-SH mode and their amplitudes decrease into the depth of $n$ periods by a factor $\left|\gamma_{\alpha}\right|^{n}$,

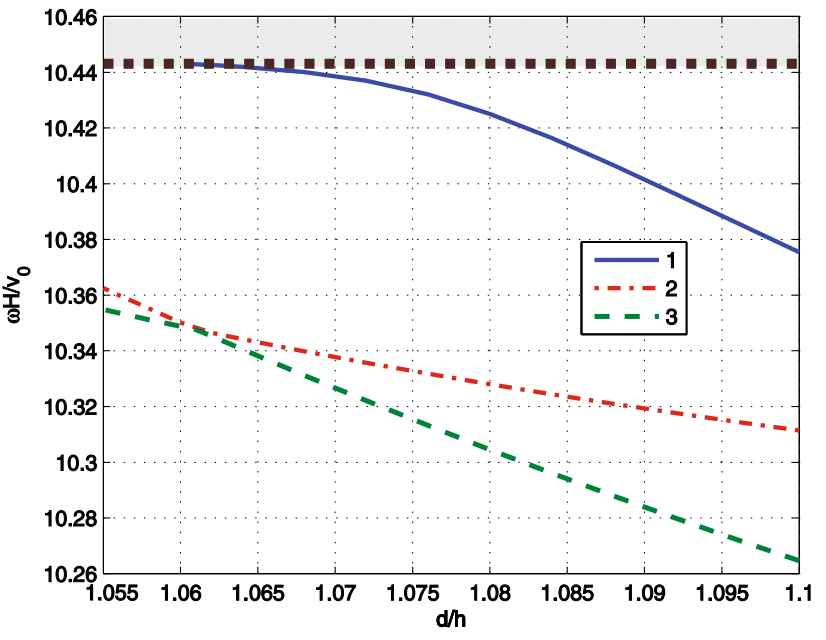

Fig. 5. Frequencies of IAWs of general polarization at $k / k_{B}=1.55$ in the bicrystal composed of the "modified" GaAs and AlAs layers vs. thickness $d$ of the defect layer. The propagation direction $X$ makes an angle of $3^{\circ}$ with the direction [100] in GaAs and AlAs layers. Curve 1 is the branch of quasiS-IAWs, curves 2 and 3 are the branch of quasiS- and quasiSH-IAWs, respectively, at $d / h>1.06$ and other way around at $d / h<1.06$. The horizontal dot line shows the edge frequency of the lowest stopband.
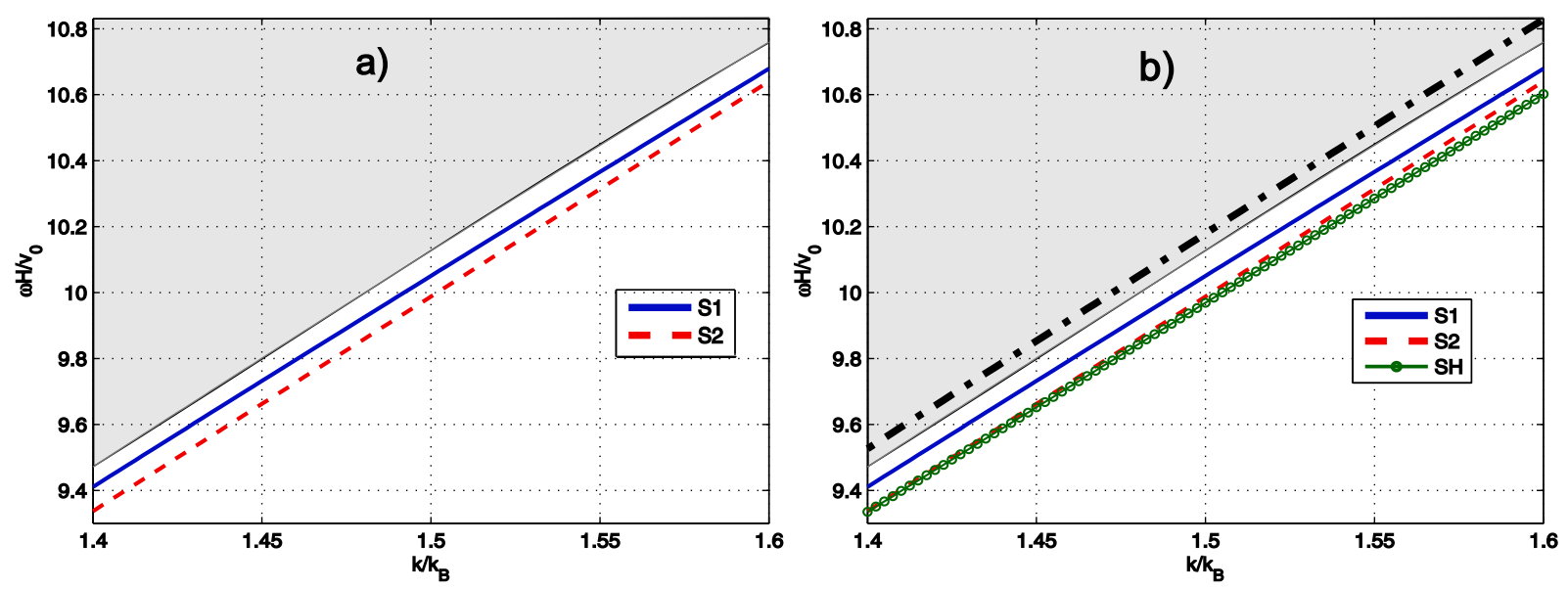

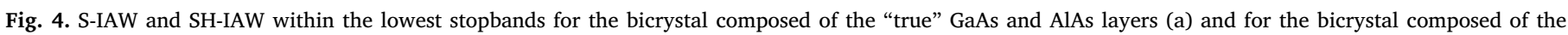

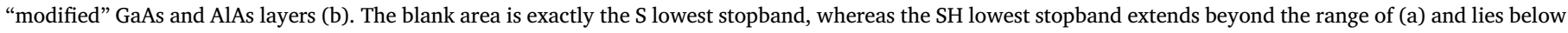
the dot-dashed line in (b). 


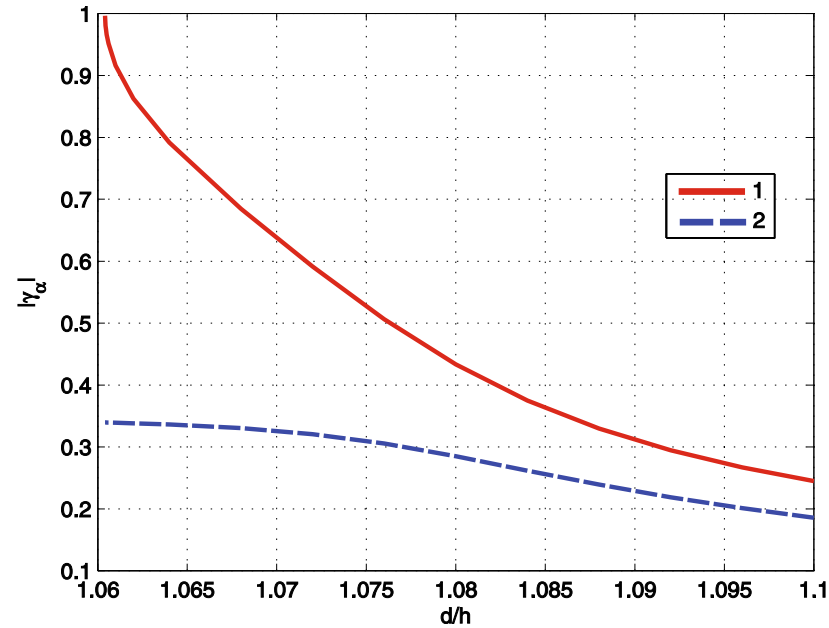

Fig. 6. Decay factors $\left|\gamma_{1}\right|=\left|\gamma_{2}\right|$ and $\left|\gamma_{3}\right|$ - of the quasiS and quasiSH partial modes of the quasiS-IAW corresponding to curve 1 in Fig. 5.

where $\gamma_{\alpha}, \alpha=1,2,3$, is the triplet of eigenvalues $\left|\gamma_{\alpha}\right|<1$ of either of the transfer matrices $\mathbf{M}^{(J)}$ of the half-infinite superlattices $J=1,2$ forming the given bicrystal (see a discussion around Eq. (39)). The evolution of the partial decay factors $\left|\gamma_{1}\right|=\left|\gamma_{2}\right|$ of the partial quasiS modes and $\left|\gamma_{3}\right|$ of the quasiSH partial mode of the given quasiS-IAW with variation of the defect layer thickness $d / h$ is shown in Fig. 6 (identity $\left|\gamma_{1}\right|=\left|\gamma_{2}\right|$ holds true owing to the crystallographic symmetry of layers). It is seen that $\left|\gamma_{1}\right|=\left|\gamma_{2}\right|$ tends to unity at $d / h \rightarrow 1.06$, which is in accordance with the behaviour of curve 1 in Fig. 5 .

A similar idea of modelling a nearly congruent pair of upper $S$ and $\mathrm{SH}$ stopbands, each with a maximum possible number (three) of mutually decoupled S-IAWs and SH-IAWs occurring in the direct and complementary bicrystals, allows us to exemplify the occurrence of the maximum number of six IAWs of general polarization, which arise as a result of a slight change of the initially symmetric propagation geometry. This goal may be achieved by taking the shear moduli $c_{44}=c_{55}$ of "modified" GaAs and AlAs to be equal to 53.2 and $50.9 \mathrm{GPa}$, that leads to almost coinciding pair of upper S and SH stopbands, whose overlap contains two SH-IAW and four S-IAW branches shared by the direct and complementary bicrystals, see Fig. 7. A perturbation of this geometry leads to the sought situation with six IAWs of general polarization in one stopband.

\section{Conclusions}

We have studied interfacial acoustic waves (IAWs) guided by the

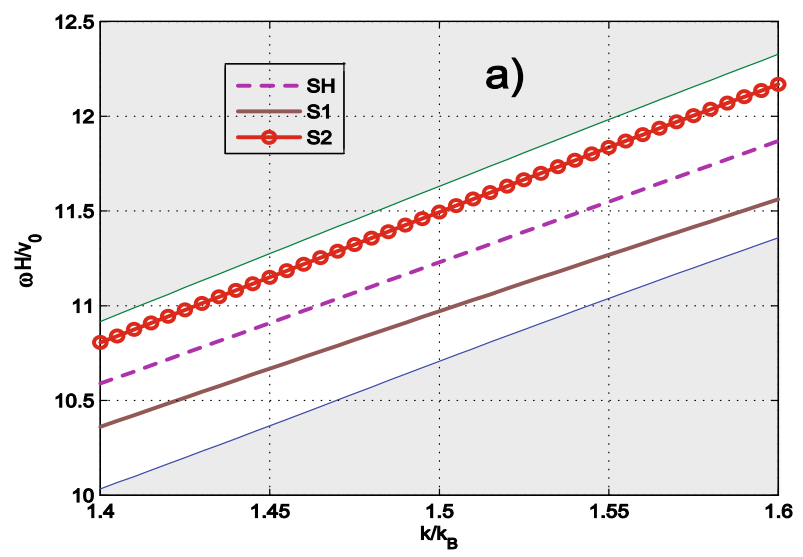

perfectly bonded interface between two half-infinite periodic superlattices. Each superlattice may consist of homogeneous or functionally graded layers of general anisotropy and may have an arbitrary assembling of its unit cell. The waves under study may be seen as an analogue of the Stoneley wave at an interface between homogeneous media, except that they are dispersive and their frequency and wavenumber form multiple branches lying in the stopband of the structure. The main objective was to establish how many IAWs can come about in a stopband. It was found that, unless the arrangement of unit cells of both superlattices of a given structure is symmetric relative to the period midplanes, the maximum possible number of IAWs at fixed wavenumber $k$ and the frequency $\omega$ varying within a stopband is three for the lowest stopband and six for any upper ones. Moreover, a stronger statement was also established which is that the maximum number of three or six IAWs per stopband is actually a bound for the total number of IAWs occurring in the given bicrystal and in its counterpart obtained by swapping its two halves (the latter transformation does not affect the band structure but leads to a different wave solutions).

The above statements can be further specialised in the case of uncoupled shear-horisontally and sagittally (SH- and S-) polarised IAWs propagating in the structures of monoclinic elastic symmetry. In this case, at most one SH-IAW at fixed $k$ is admitted in the lowest SHstopband of the direct and complementary bicrystals in total, i.e. there may be no such waves or there may be a single one which emerges in either the direct or complementary bicrystal. At most two S-IAWs can occur in the lowest S-stopband of the direct and complementary bicrystals in total. Regarding upper SH- and S-stopbands, up to two SHIAWs and four S-IAWs can exist per such a band in the direct and complementary bicrystals in total.

It is noteworthy that the aforementioned upper bounds for the number of IAWs of general polarisation as well as for that of SH-IAWs and S-IAWs are attainable. This is confirmed by numerical computations of IAWs guided by a defect layer in a periodically bilayered structure. It is certainly understood that the maximum number does not at all has to be reached in an arbitrary case. Generally speaking, the occurrence of IAWs is more probable when one and/or the other superlattices constituting a bicrystal admits a surface-wave solution on its clamped boundary, i.e. when the impedance matrix has a pole. In particular, there is a transparent and relatively definitive link between the existence of IAWs and the impedance poles in the case of uncoupled SH-waves.

\section{Declaration of Competing Interest}

The authors declare that they have no known competing financial interests or personal relationships that could have appeared to influence the work reported in this paper.

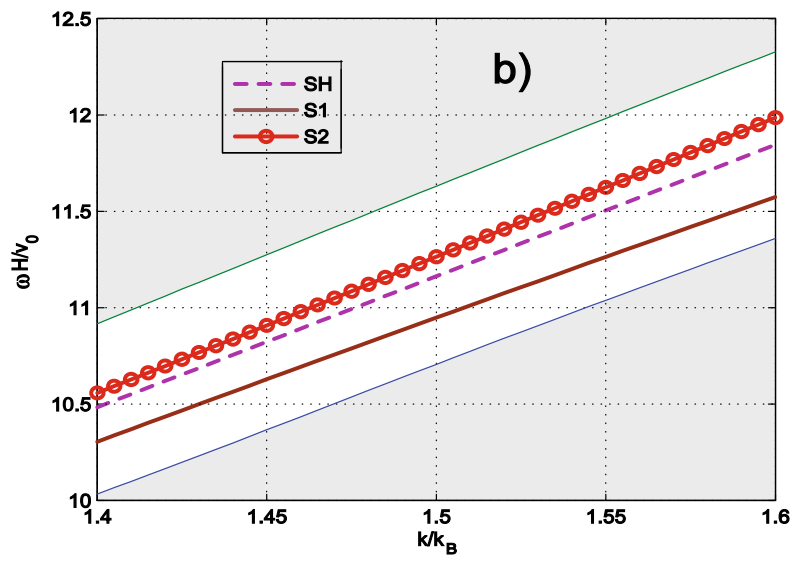

Fig. 7. S-IAW and SH-IAW dispersion branches in the direct (a) and complementary (b) bicrystals composed of the "modified" GaAs and AlAs layers. Blank area is the overlap of SH and S upper stopbands. 


\section{Acknowledgements}

The authors thank V. I. Alshits for helpful discussions. The work of A.D. was supported by the Ministry of Science and Higher Education of the Russian Federation within the State assignment FSRC "Crystallography and Photonics" RAS.

\section{References}

[1] B.A. Auld, Acoustic Fields and Waves in Solids vol. I,II, Krieger Publishing Company, 1990.

[2] P.A. Deymier PA (Ed.), Acoustic Metamaterials and Phononic Crystals, Springer, Berlin/Heidelberg, Germany, 2013.

[3] V. Laude, Phononic crystals: artificial crystals for sonic, acoustic, and elastic waves. De Gruyter Studies in Mathematical Physics, vol. 26, Walter De Gruyter, Berlin, Germany, 2015

[4] A. Khelif, A. Adibi (Eds.), Phononic Crystals: Fundamentals and Applications, Springer, Berlin/Heidelberg, Germany, 2016.

[5] R.E. Camley, B. Djafari-Rouhani, L. Dobrzynski, A.A. Maradudin, Transverse elastic waves in periodically layered infinite and semi-infinite media, Phys. Rev. B 27 (1983) 7318-7329, https://doi.org/10.1103/PhysRevB.27.7318.

[6] B. Djafari-Rouhani, L. Dobrzynski, O. Hardouin Dupac, R.E. Camley, A.A. Maradudin, Sagittal elastic waves in periodically layered infinite and semiinfinite superlattices, Phys. Rev. B 28 (1983) 1711-1720, https://doi.org/10.1103/ PhysRevB.28.1711.

[7] B. Djafari-Rouhani, A.A. Maradudin, R.F. Wallis, Rayleigh waves on a superlattice stratified normal to the surface, Phys. Rev. B 29 (1984) 6454-6462, https://doi. org/10.1103/PhysRevB.29.6454.

[8] A.N. Podlipenets, N.A. Shul'ga, Numerical study of Rayleigh and Lamb waves in orthotropic periodic structures, Int. Appl. Mech. 23 (1987) 1013-1017.

[9] A. Nougaoui, B. Djafari-Rouhani, Elastic waves in periodically layered infinite and semiinfinite anisotropic media, Surf. Sci. 185 (1987) 125-153, https://doi.org/10. 1016/S0039-6028(87)80618-0.

[10] E.H. El Boudouti, B. Djafari-Rouhani, A. Nougaoui, Surface elastic waves in superlattices: sagittal localized and resonant modes, Phys. Rev. B 51 (1995) 13801-13804, https://doi.org/10.1103/PhysRevB.51.13801.

[11] T. Aono, S. Tamura, Surface and pseudosurface acoustic waves in superlattices, Phys. Rev. B 58 (1998) 4838-4845, https://doi.org/10.1103/PhysRevB.58.4838.

[12] Ph. Gatignol, C. Potel, J.F. de Belleval, Two families of modal waves for periodic structures with two field functions: a Cayleigh-Hamilton approach, Acustica-Acta Acustica 93 (2007) 959-975.

[13] S.-Y. Ren, Y.-C. Chang, Theory of confinement effects in finite one-dimensional phononic crystals, Phys. Rev. B 75 (2007) 212301, https://doi.org/10.1103/ PhysRevB.75.212301.

[14] A.L. Shuvalov, O. Poncelet, S.V. Golkin, Existence and spectral properties of shear horizontal surface waves in vertically periodic half-spaces, Proc. R. Soc. Lond. A 465 (2009) 1489-1511, https://doi.org/10.1098/rspa.2008.0457.

[15] A.N. Norris, A.L. Shuvalov, A.A. Kutsenko, The matrix sign function for solving surface wave problems in homogeneous and laterally periodic elastic half-spaces, Wave Motion 50 (2013) 1239-1250, https://doi.org/10.1016/j.wavemoti.2013.03. 010.

[16] E.H. El Boudouti, B. Djafari-Rouhani, One-dimensional phononic crystals, in: P.A. Deymier, (ed.), Acoustic Metamaterials and Phononic Crystals, Springer, Berlin/ Heidelberg, Germany, 2013, pp. 45-94.

[17] E.H. El Boudouti, B. Djafari-Rouhani, E.M. Khourdifi, L. Dobrzynski, Surface and interface elasticwaves in superlattices: transverse localized and resonant modes, Phys. Rev. B 48 (10) (1993) 10 987-10 997, https://doi.org/10.1103/PhysRevB.48. 10987.

[18] E.H. El Boudouti, B. Djafari-Rouhani, A. Akjouj, L. Dobrzynski, Theory of surface and interface transverse elastic waves in N-layer superlattices, Phys. Rev. B 54 (1996) 14728-14741, https://doi.org/10.1103/PhysRevB.54.14728.
[19] A. Bousfia, E.H. El Boudouti, D. Bria, A. Nougaoui, Surface and interface shear horizontal acoustic waves in piezoelectric superlattices, J. Appl. Phys. 87 (2000) 4507-4513, https://doi.org/10.1063/1.373097.

[20] B.J. Lee, A.C. To, Enhanced absorption in one-dimensional phononic crystals with interfacial acoustic waves, Appl. Phys. Lett. 95 (2009) 031911, https://doi.org/10 1063/1.3182819.

[21] N. Liu, J. Yang, Z.H. Qian, S. Hirose, Interface waves in functionally graded piezoelectric materials, Int. J. Eng. Sci. 48 (2010) 151-159, https://doi.org/10.1016/j ijengsci.2009.07.008.

[22] K.A. Ingebrigtsen, A. Tonning, Elastic surface waves in crystals, Phys. Rev. 184 (1969) 942-951, https://doi.org/10.1103/PhysRev.184.942.

[23] D.M. Barnett, J. Lothe, Considerations of the existence of surface (Rayleigh wave) solutions in anisotropic elastic crystals, J. Phys. F 4 (1974) 1618-1635, https://doi org/10.1088/0305-4608/4/10/010.

[24] P. Chadwick, P.K. Currie, Stoneley waves at an interface between elastic crystals, J. Mech. Appl. Math. 27 (1974) 497-503, https://doi.org/10.1093/ajmam/27.4.497.

[25] J. Lothe, D.M. Barnett, On the existence of surface wave solutions for anisotropic halfspaces with free surface, J. Appl. Phys. 47 (1976) 428-433, https://doi.org/10 1063/1.322665.

[26] P. Chadwick, G.D. Smith, Foundation of the theory of surface waves in anisotropic elastic media, Adv. Appl. Mech. 17 (1977) 303-376.

[27] D.M. Barnett, J. Lothe, S.D. Gavazza, M.J.P. Musgrave, Considerations of the ex istence of interfacial (Stoneley) waves in bonded anisotropic elastic half-spaces, Proc. R. Soc. Lond. A 402 (1985) 153-166, https://doi.org/10.1098/rspa.1985. 0112.

[28] D.M. Barnett, J. Lothe, Free surface (Rayleigh) waves in anisotropic media: the surface impedance method, Proc. R. Soc. Lond. A 402 (1985) 135-152, https://doi org/10.1098/rspa.1985.0111.

[29] D.M. Barnett, S.D. Gavazza, J. Lothe, Slip waves along the interface between two anisotropic elastic half-spaces in sliding contact, Proc. R. Soc. Lond. A 415 (1988) 389-419, https://doi.org/10.1098/rspa.1988.0020.

[30] A.N. Darinskii, A.L. Shuvalov, Surface acoustic waves on one-dimensional phononic crystals of general anisotropy: existence considerations, Phys. Rev. B 98 (2018) 024309, https://doi.org/10.1103/PhysRevB.98.024309.

[31] A.N. Darinskii, A.L. Shuvalov, Existence of surface acoustic waves on half-infinite one-dimensional piezoelectric phononic crystals of general anisotropy, Phys. Rev. B 99 (2019) 174305, https://doi.org/10.1103/PhysRevB.99.174305.

[32] A.N. Darinskii, A.L. Shuvalov, Non-leaky surface acoustic waves in the passbands of one-dimensional phononic crystals, Ultrasonics 98 (2019) 108-118, https://doi org/10.1016/j.ultras.2019. 06.002.

[33] A.N. Darinskii, A.L. Shuvalov, Surface acoustic waves in one-dimensional piezoelectric phononic crystals with symmetric unit cell, Phys. Rev. B 100 (2019) 184303, https://doi.org/10.1103/PhysRevB.100.184303.

[34] A.N. Darinskii, A.L. Shuvalov, Interfacial acoustic waves in one-dimensional anisotropic phononic bicrystals with a symmetric unit cell, Proc. R. Soc. A 475 (2019) 20190371, https://doi.org/10.1098/rspa.2019.0371.

[35] A.N. Stroh, Steady state problems in anisotropic elasticity, J. Math. Phys. 41 (1962) 77-103, https://doi.org/10.1002/sapm196241177.

[36] T.C.T. Ting, Anisotropic Elasticity: Theory and Applications, Oxford University Press, Oxford, UK, 1996.

[37] Y. Fu, Hamiltonian interpretation of the Stroh formalism in anisotropic elasticity, Proc. R. Soc. A 463 (2007) 3073-3087, https://doi.org/10.1098/rspa.2007.0093.

[38] M.C. Pease III, Methods of Matrix Algebra, Academic Press, New York, NY, 1965.

[39] A.M.B. Braga, G. Herrmann, Floquet waves in anisotropic periodically layered composites, J. Acoust. Soc. Am. 91 (1992) 1211-1227, https://doi.org/10.1121/1 402505.

[40] A.L. Shuvalov, A.A. Kutsenko, M.E. Korotyaeva, O. Poncelet, Love waves in a coated vertically periodic substrate, Wave Motion 50 (2013) 809-820, https://doi.org/10 1016/j.wavemoti.2013.02.005.

[41] A.P. Silin, Semiconductor superlattices, Usp. Fiz. Sci. 147 (1985) 485 [Sov. Phys. Usp. 28, 972 (1985)].

[42] http://www.matprop.ru/. 